\title{
Feasibility of a respiratory nurse specialist- led sleep disorder service in a district general hospital
}

DM Comer ${ }^{1}$

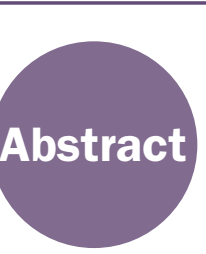

Aim The burden of referrals to investigate sleep disorders has escalated considerably. In order to address this, the feasibility of a nurse-led sleep disorder clinic was investigated.

Methods Sleep referrals were stratified on the basis of information contained within the primary care referral, and appropriate home-based sleep studies arranged if obstructive sleep apnoea was considered the most likely working diagnosis. Selected patients were started on continuous positive airway pressure treatment if deemed appropriate. Education was almost exclusively provided by specialist nursing staff or by means of validated information by the British Lung Foundation.

Correspondence to:

DM Comer

Department of Respiratory

Medicine

Altnagelvin Hospital

Londonderry BT47 6SB

Northern Ireland

Email:

dcomer@doctors.org.uk

Results Eighty patients were included in the respiratory nurse-led sleep service. Feedback from the patient questionnaire was positive and 59 patients were managed without requirement to attend consultant-led outpatient services. Non-sleep breathing disorders as the reason for daytime somnolence, and patients requiring referral to tertiary services, were identified.

Conclusion Developing a respiratory nurse-led sleep service is adequate to identify non-sleep breathing disorders, is fit for purpose, functions broadly in accordance with American Sleep Society guidelines, and is well received by patients.

Keywords: nurse-led, sleep apnoea

Declaration of interests: No conflict of interests declared

\section{Introduction}

Referrals from across multiple specialities to assess for sleep disordered breathing have increased in an exponential fashion, ${ }^{1}$ and sleep services are struggling to meet the relentless increment in demand. Nurse-led care in other chronic medical conditions has been demonstrated with favourable outcomes, both in terms of patient-centred endpoints and quality of care when compared with relevant guidelines. ${ }^{2,3}$ As far as is known, similar studies have not been undertaken in sleep disorders; perhaps a patient group where there would be a reasonable expectation to anticipate disproportionate benefits in adopting such an approach.

It is with this background in mind that the feasibility of a respiratory nurse specialist(RNS)-led sleep service was assessed, with consultant input and supervision. This service was managed in such a way as to be more selective in those patients selected for review at consultant-led outpatient (CLO) services. The purpose was primarily to reduce waiting times from initial referral to intervention, while maintaining a service that functioned in accordance with the American Association of Sleep Medicine Guidelines (AASM), and mitigating the overall burden on CLO services.

The health consequences of sleep apnoea are diverse, including, but not restricted to, disorders of emotional state, ${ }^{4}$ cognition, ${ }^{5}$ immune defence ${ }^{6}$ and cardiovascular health. ${ }^{7}$ Furthermore, individuals with obstructive sleep apnoea have a heightened risk of car accidents. ${ }^{8}$ However, many of these adverse consequences can be addressed by treatment of the sleep disorder itself, serving to mitigate the adverse effects outlined above..$^{9,10}$ It is with this in mind that the benefits of timely intervention are obvious.

\section{Methods}

Sleep referrals were stratified on the basis of information contained within the primary care referral, and appropriate home-based sleep studies were arranged prior to clinical assessment if sleep disordered breathing was deemed to be the most likely final diagnosis. Patients were informed by post of the rationale for the test. Depending on the result, 
patients were subsequently managed by the RNS, with an interest in sleep disordered breathing, or a CLO appointment was arranged.

The RNS discussed appropriate interventions with patients, and they were referred back to outpatient services in the event of an inadequate response to continuous positive airway pressure (CPAP) treatment. Advice on driving regulations, lifestyle, and the importance of CPAP treatment was outlined almost exclusively by specialist nursing staff, or by means of validated information by post. Patient satisfaction was determined by sending a questionnaire to a random selection of 20 patients in order to assess a range of domains deemed most relevant (Appendix 1). The outcome of the RNS-led service was compared with historical referrals managed in the conventional fashion, with initial assessment at CLO clinics.

Differences in endpoints between both services were determined using Fishers exact testing. Relevant endpoints were considered to be patient numbers started on CPAP, numbers referred to tertiary services for consideration of polysomnography, those with an alternative explanation identified for daytime somnolence, and the proportion not attending appointments in each pathway.

\section{Results}

Eighty patients were included in the RNS-led sleep service. An outline of how this group of patients passed through the virtual sleep service is shown in Appendix 2. Thirtynine subjects had sufficient sleep disordered breathing evident on home-based studies, with typical symptoms and elevated Epworth Sleepiness Scale scores to clearly warrant intervention. Although these subjects did not attend CLO clinics, all continue to be followed up by respiratory nurses to ensure adequate resolution of symptoms and to provide relevant education.

Overall, 59 of the 80 patients were managed without attending CLO clinics, although three, who were initially started on CPAP and without an initial outpatient assessment, subsequently did not respond adequately to treatment. These individuals were later assessed at a CLO clinic. Of the 80 patients, two were considered on the basis of the history and home-based sleep studies to warrant investigation for narcolepsy. One had predominantly central sleep disordered breathing. Of the 80 patients, six were considered to have an alternative explanation for their symptoms (circadian rhythm disorder (four), poor sleep hygiene (one) and suspected depression (one)). Responses to the questionnaire are shown in Appendix 3.

Outcomes for sleep referrals managed in the conventional fashion, with an initial assessment at the CLO clinic, and without a sleep test result, which were determined in a retrospective fashion, are shown in Appendix 4. 'Did not attend' rates were similar for both services, as was the requirement for multichannel sleep studies, suggesting that resource utilisation was similar. Those subjects with an alternative medical explanation were identified more promptly, but this represented a small number in both pathways (Appendices 2 and 4). There were no statistical differences in the proportion of patients started on CPAP, referred for polysomnography, those having an alternative explanation identified for their symptoms, and those failing to attend appointments (Appendix 5).

Average waiting time for CLO assessment, for conventional pathways, was approximately 14 months. This is a consequence of the burden of sleep referrals primarily from primary care and ENT services, with smaller numbers from neurology and endocrinology. For non-urgent cases, the interval from establishing a diagnosis of sleep disordered breathing to starting CPAP was six weeks in both pathways. Therefore, identifying subjects who could be effectively and safely managed without consultant input significantly reduced waiting times and workload on CLO services.

\section{Discussion}

A RNS-led sleep service appears to be both effective and justified, conferring a more rational approach to the delivery of care to patients with suspected sleep apnoea. We have shown, in this relatively small group of patients, that by designing pathways with careful consideration of referral information, and ensuring adequate support to specialist nursing staff, a more efficient and equally functional service is provided. Patient satisfaction with the service was noticeable.

Keeping in mind that a timely intervention with CPAP improves quality of life and psychological profile in individuals with obstructive sleep apnoea, ${ }^{11}$ the benefits of a more rational approach are clear. Waiting time to start CPAP, considered the appropriate intervention for the patients included with positive studies and elevated Epworth scores, was substantially reduced for this group of patients. Follow up, provided exclusively by a RNS, ensured a satisfactory resolution of symptoms and that adequate control of the obstructive sleep apnoea itself was achieved. The latter was complemented by remote monitoring. Historical delays before assessment at CLO clinics were around 14 months, and this was removed for this particular group of patients.

A RNS-led service does incur a degree of risk. The AASM indicates that all such patients should be assessed by a sleep specialist. Perhaps the most significant concern would be the potential to 'miss' disorders leading to daytime somnolence, such as idiopathic hypersomnolence, periodic limb movement disorder, narcolepsy, chronic heart failure, depression or circadian rhythm disorders. If this were to occur, the possible risk lies in the potential to inappropriately intervene with CPAP. However, it could be speculated that this risk is relatively low. All patients with home-based studies which were normal, or almost normal with only trivial abnormalities, were evaluated at CLO clinics to assess for the latter mentioned conditions. It also could be argued that any given patient could have sleep disordered breathing 
which was not the cause of their symptoms and merely an incidental finding. This is particularly pertinent keeping in mind sleep disordered breathing affects up to $24 \%$ of the adult population. ${ }^{12}$ This risk could be adequately offset by ongoing review by RNS staff, which itself serves to mitigate this inherent risk.

Three of the 80 patients included had either normal or trivial abnormalities on home-based multichannel sleep studies. These patients were referred to tertiary services for assessment for narcolepsy, all of whom had other suggestive symptoms for this to be considered a feasible diagnosis. Considering the incidence of narcolepsy in our region to be in the order of $0.05 \%$, as indicated on the Narcolepsy UK website, this referral rate seems to be appropriate.

Remote monitoring was used to further reduce the demands on the service. Surrogates for Apnoea-Hypopnoea Index, derived from the CPAP hardware, provided a measure of control of sleep disordered breathing, and were obtained by means of downloading electronic information from an electronic chip within the hardware. This was delivered to and from our centre by post. This estimate of Apnoea-Hypopnoea Index was complemented by patient self-scoring on the Epworth Sleepiness Scale, and these results, taken together, allowed a judgment to be made as to levels of control of sleep apnoea. To the best of the author's knowledge, there are no published data in the scientific literature to substantiate the validity of the output of the CPAP hardware in terms of control of sleep disordered breathing. With this in mind, the interpretation of these findings was tempered with a degree of caution and clinical judgement.

A RNS-led sleep disordered breathing service, started by investigations organised on the basis of information within the referral from primary care, reduced waiting times considerably. Pathways to identify selected subjects for outpatient assessment were successful. Appropriate proportions appear to have been identified in order to be referred for more detailed sleep inpatient assessments. These findings at least suggest that rationalising a sleep service in this fashion maintains a service that is adequate to identify non-sleep disorder breathing pathology, is fit for purpose, functions broadly in accordance with AASM guidelines, and is well received by patients.

Driving regulations from the DVLA have recently changed, and, in parts, are somewhat open to interpretation. One patient, who was effectively managed along the RNS sleep service pathway, required at a later date to be reviewed at a CLO service, after a request from the driving authorities prior to reinstating their driving licence. Another patient requested further clarification on the regulations, and attended a CLO clinic for this purpose. In terms of resource utilisation, this negated the benefit accrued from the RNS service, in so far that had these particular patients been assessed at the outset at CLO services, the latter requirement for reassessment may not have been as pertinent.

The inherent disadvantages of such an approach, and the measurable risks of incurring a delay in the diagnosis of potential non-sleep disordered breathing pathology, are understood. However, a more rational sleep service has the potential to reduce the burden on CLO services by a factor of 4 , and reduces the interval to starting CPAP treatments when deemed to be clearly indicated. In order to meet the heightened demands for these services, it should be agreed that a more balanced and considered approach is warranted for those individuals with sleep disorders and this must be done in a meaningful and strategic way. This needs to be implemented in a substantial, sustained, and consistent fashion in order to be successful, and to ultimately help combat the epidemic of sleep breathing disorders. (1)

\section{Appendices}

Appendices 1-5 are available with the online version of this paper, which can be accessed via http://www.rcpe.ac.uk/ journal, and selecting issue $47 / 2$.

\section{References}

1 Crummy F, Piper AJ, Naughton MT. Obesity and the lung: 2. Obesity and sleep-disordered breathing. Thorax 2008; 63: 738-46.

2 Sharples LD, Edmunds J, Bilton D et al. A randomised controlled crossover trial of nurse practitioner versus doctor led outpatient care in a bronchiectasis clinic. Thorax 2002; 57: 661-6.

3 Loftus LA, Weston V. The development of nurse-led clinics in cancer care. J Clin Nurs 2001; 10: 215-20.

$4 \mathrm{Ji}$ XW, Chan CHY, Lau BHP et al. The interrelationship between sleep and depression: a secondary analysis of a randomized controlled trial on mind-body-spirit intervention. Sleep Med 2017; 29: 41-6.

5 Harmell AL, Neikrug AB, Palmer BW et al. Obstructive sleep apnea and cognition in Parkinson's disease. Sleep Med 2016; 21: 28-34.

6 Wilder A, Mustafa FB, Earnest A et al. Impact of partial sleep deprivation on immune markers. Sleep Med 2013; 14: 1031-4.

7 Shah NA, Yaggi HK, Concato J et al. Obstructive sleep apnea as a risk factor for coronary events or cardiovascular death. Sleep Breath 2010; 14: 131-6.
8 McNicholas WT, Rodenstein D. Sleep apnoea and driving risk: the need for regulation. Eur Respir Rev 2015; 24: 602-6.

9 Lévy P, Bonsignore MR, Eckel J. Sleep, sleep-disordered breathing and metabolic consequences. Eur Respir J 2009; 34: 243-60.

10 Buchner NJ, Quack I, Stegbauer J et al. Treatment of obstructive sleep apnea reduces arterial stiffness. Sleep Breath 2012; 16 : 123-33.

11 Diamanti C, Manali E, Ginieri-Coccossis M. Depression, physical activity, energy consumption, and quality of life in OSA patients before and after CPAP treatment. Sleep Breath 2013; 17: 1159-68.

12 Young T, Palta M, Dempsey J et al. The occurrence of sleepdisordered breathing among middle-aged adults. $N$ Engl J Med 1993; 328: 1230-5. 